\title{
Optogenetic Stimulation of Primary Cardiomyocytes Expressing ChR2
}

\author{
Hoda Keshmiri Neghab ${ }^{1,2}{ }^{\circledR}$, Mohammad Hasan Soheilifar ${ }^{3,4}{ }^{\oplus}$, Ali Akbar Saboury ${ }^{(\oplus}$, Bahram Goliaei ${ }^{\circledR}$, Jun \\ Hong $^{(\mathbb{E}}{ }^{\text {, Gholamreza Esmaeeli Djavid }}{ }^{* \mathbb{D}}$
}

${ }^{1}$ Department of Photo Healing and Regeneration, Medical Laser Research Center, Yara Institute, ACECR, Tehran, Iran ${ }^{2}$ Institutes of Biochemistry and Biophysics (IBB), University of Tehran, Tehran, Iran

${ }^{3}$ Department of Medical Laser, Medical Laser Research Center, Yara Institute, ACECR, Tehran, Iran

${ }^{4}$ Research Center for Molecular Medicine, School of Medicine, Hamadan University of Medical Sciences, Hamadan, Iran ${ }^{5}$ School of Life Sciences, Henan University, China

\section{*Correspondence to} Gholamreza Esmaeeli Djavid, Department of Photo Healing and Regeneration, Medical Laser Research Center, Yara Institute, ACECR, Tehran, Iran.

Email: djavid@ut.ac.ir

Received: June 28, 2020 Accepted: December 2, 2020 epublished: July 4, 2021

\begin{abstract}
Introduction: Non-clinical cardiovascular drug safety assessment is the main step in the progress of new pharmaceutical products. Cardiac drug safety testing focuses on a delayed rectifier potassium channel block and QT interval prolongation, whereas optogenetics is a powerful technology for modulating the electrophysiological properties of excitable cells.

Methods: For this purpose, the blue light-gated ion channel, channelrhodopsin-2 (ChR2), has been introduced into isolated primary neonatal cardiomyocytes via a lentiviral vector. After being subjected to optical stimulation, transmembrane potential and intracellular calcium were assessed. Results: Here, we generated cardiomyocytes expressing ChR2 (light-sensitive protein), that upon optical stimulation, the cardiomyocytes depolarized result from alterations of membrane voltage and intracellular calcium.

Conclusion: This cell model was easily adapted to a cell culture system in a laboratory, making this method very attractive for therapeutic research on cardiac optogenetics.

Keywords: Primary cardiomyocyte; Optogenetics; ChR2.
\end{abstract}

\section{Introduction}

Heart arrhythmias are described as any change in the normal rhythms of the heart's electrical system and are the leading causes of worldwide mortality. Cardiac arrhythmias can be categorized as a slow heartbeat (bradyarrhythmias) involving the implantation of a pacemaker, versus a fast-beating rate (tachyarrhythmias) requiring the implantable cardiac defibrillators and antiarrhythmic drugs. ${ }^{1}$ Preclinical evaluation of drug cardiotoxicity concentrates on the in vitro block of the rapid delayed rectifier current that conducts $\left(\mathrm{K}^{+}\right)$, combined with in vivo QT prolongation for cardiotoxicity assessment. ${ }^{2}$ Optogenetics has created a new opportunity for drug screening, particularly in drug discovery. Optogenetic tools provide a way of probing the electrophysiological responses of cardiomyocytes in drug efficacy and safety testing. ${ }^{3}$

Optogenetics needs light-activated protein, which naturally occurs or is chemically modified ${ }^{4}$. The most commonly used opsin, channelrhodopsin-2 (ChR2) is the light-gated ion channel, originated from algae, and has emerged as a new widespread excitatory tool not only in neuroscience but also in cardiology. ${ }^{5}$ In the present study, we generated cardiomyocytes expressing an improved ChR2 that is activated by blue light stimulation. Following photon absorption, the chromophore retinal, which covalently binds to the channel and serves as a lightsensing element, photoisomerizes. This isomerization triggers the opening of the ion channel allowing the passage of cations across the cell membrane according to their electrochemical gradient. Therefore, we generated a population of cardiomyocytes in a culture that is stimulated by light with both spatial and temporal precision. This cell culture model can be utilized in cardiac research.

\section{Methods \\ Neonatal Rat Cardiomyocyte Isolation and Characterization \\ Primary cardiomyocytes were isolated from neonatal rats according to the protocol optimized before. ${ }^{6}$ Briefly, cardiac tissues were digested with $0.025 \%$ trypsin/ EDTA and then the supernatant was carefully collected and centrifugated at $1800 \mathrm{rpm}$ for 20 minutes. The pellet was suspended with DMEM, 10\% FCS, $1 \%$ non-}


essential amino acid, and 1\% L-glutamine. After loading in a percoll gradient and centrifugation at $6000 \mathrm{rpm}$ for 30 minutes, the cell pellets were suspended in a culture medium and incubated for 1.5-2 hours in a humidified, $37^{\circ} \mathrm{C}$ and $5 \% \mathrm{CO}_{2}$ incubator to allow the attachment of non-myocardial cells to the $0.1 \%$ gelatin-coated flask. The supernatant fluid that contains non-adhesive myocytes was centrifuged at $1800 \mathrm{rpm}$ for 20 minutes. In the end, the cardiomyocytes were cultured and placed into the incubator. To characterize neonatal rat derived cardiomyocytes in cell culture, we used the alpha-actinin antibody and western blot technique.

\section{Lentiviral Transduction of Cardiomyocytes}

To generate cell-type-specific expression of ChR2 in isolated cardiomyocytes, we used pcDNA3.1/hChR2 (H134R)-eYFP kindly given by the group of professor Karl Deisseroth (Addgene plasmid \#20940). The lentivirus system was generated by co-transfection of transfer plasmid LV-105 harboring ChR2 (GeneCopia) with the packaging vector pLV-HELP (InvivoGen) and envelope plasmid pMD2G (InvivoGen), into HEK293T cell culture. Viral vectors, which were released into the culture medium, were purified with Amicon Ultra-4 centrifugal filter units (Millipore, USA) and stored at $-80^{\circ} \mathrm{C}$ for further usage.

\section{Light Stimulation, Voltage, and Calcium Imaging}

For optical stimulation and intensity quantification, we used a multi-mode fluorescence microplate reader (Biotek Synergy H4 hybrid) in an endpoint kinetic mode, which stimulates each microplate well at $470 \mathrm{~nm}$ (intensity 1 $\mathrm{mW} / \mathrm{cm}^{2}$ ). To determine the membrane potential and intracellular $\mathrm{Ca}^{2+}$ concentration, voltage fluorescent dye DiANEPPS (Santa Cruz Biotechnology, sc-214873) and fluorescent calcium indicator, Fura-2 (Abcam, ab120837) respectively are used. Optical imaging of transduced cells was performed using a fluorescence microscope (Zeiss, Germany).

\section{Results \\ Characterization of Primary Cultured Neonatal Rat Cardiomyocytes}

Preparation of high purity and quality primary cardiomyocytes is important for successful experiments in the field of cardiology. As shown in Figure 1, isolated cardiomyocytes are viable and rod-shaped. Sarcomeric $a$-actinin commonly occurs at the Z-disc of sarcomeres in cardiomyocytes. Sarcomeric $\alpha$-actinin was present in both the positive control and samples from cardiomyocytes (Figure 1b).

\section{Detection of ChR2 Expression in Cardiomyocytes}

We first evaluated $\mathrm{ChR} 2$ expression efficiency in cardiomyocytes with an eYFP fluorescent reporter. By visualizing the expression of ChR2 using the eYFPencoding vector, we demonstrated strong and stable transgene expression in cardiomyocytes (Figure 2a).

In this investigation, we presumed that the eYFP indicator intensity is equivalent to ChR2 expression; thus, the increases shown in Figure $2 \mathrm{~b}$ demonstrate ChR2 expression in the membrane. Quantification of the fluorescence intensity of CM-ChR2 was 2.5 -fold $(P<$ $0.05)$ compared to CM. Due to the loss of eYFP protein on a mock vector, fluorescent emission was not seen in the virus control.

\section{Electrophysiology Features of Cardiomyocyte Expressing ChR2}

We evaluated the electrophysiological characteristic of CMs that express ChR2 compared with CMs. By using the optical potentiometric fluorescent probes, such as di-8-ANEPPS and Fura-2, we observed that fluorescence intensity in CM-ChR2 significantly increased after blue light stimulation compared with the unstimulated group. It means that following blue light stimulation, the potential difference across the membrane decreased and intracellular calcium level increased compared with the unstimulated group.
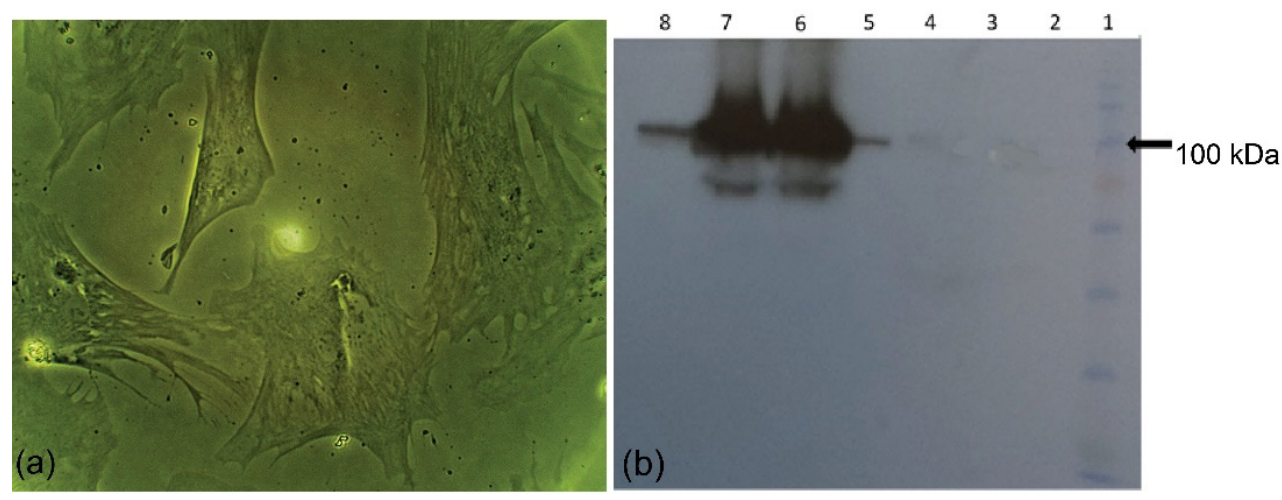

Figure 1. (a) Cell morphology of primary cultured neonatal rat cardiomyocyte under an optical microscope. (b) Expression of $\alpha$-actinin; Lane 1: 11-242 kDa ladder (Sinaclon Co. Iran), Lane 2-4: Negative control, Lanes 5 and 8: extracted protein from isolated cardiomyocytes $(100 \mathrm{kDa})$, Lanes 6 and 7: positive control, extracted protein from the isolated heart. 


\section{Discussion}

In our study, cardiomyocytes derived from neonatal rats were easily transducted with the ChR2 construct so they could be studied as an in vitro model system. Druginduced cardiotoxicity is a major adverse effect associated with pharmaceutical development. ${ }^{7,8}$ Presently, estimating the cardiotoxicity potential of therapeutic drugs is a critical parameter in novel drug development, and various models have been established to help its anticipation. One of the in vitro applications of cardiac optogenetics is the possibility of investigation of electrophysiological properties of cardiomyocyte monolayers under different conditions. This approach may be useful in studies of drug efficacy and induced cardiac side effects. However, the main disadvantage of electrical stimulation is the production of stimulation artifacts that prevent the concurrent recording of electrical potentials produced by a cell. Optogenetic stimulation of excitable cells prepares an alternative way for the investigation of cellular-level electrophysiological interrogation. For instance, Klimas et al introduced an automated platform to quantify action potential and calcium transmission, which are an indicator of cardiac toxicity. ${ }^{9}$

Optopatch is a platform that enables concurrent optical stimulation and optical imaging. Optopatch combines the Channelrhodopsin actuator CheRiff and fluorescence red (a)

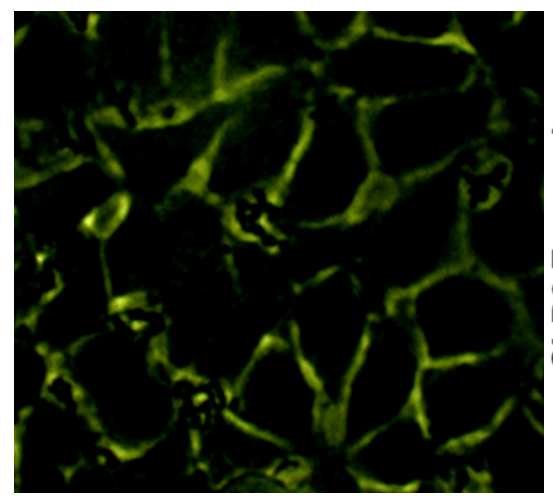

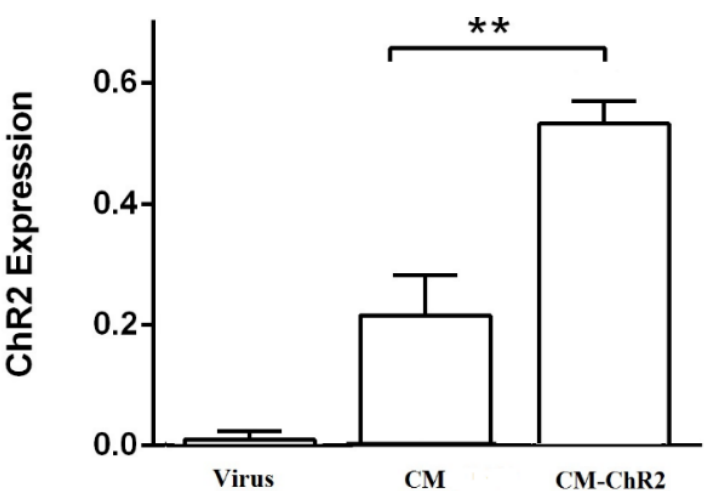

(b)

Figure 2. Detection of ChR2 Expression. (a) Fluorescence microscopy of eYFP-ChR2 expression, localized in the membrane in CMChR2. (b) Measurement of the fluorescence intensity of eYFP-ChR2 expression.

(a)
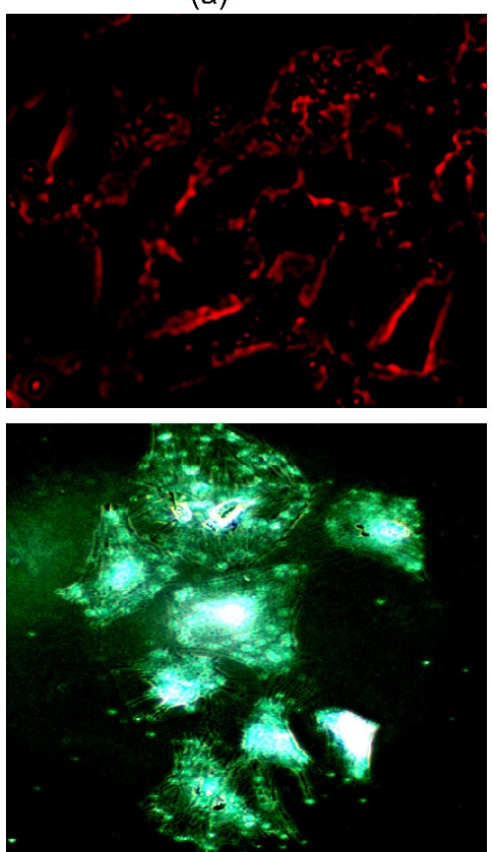

(c) (b)
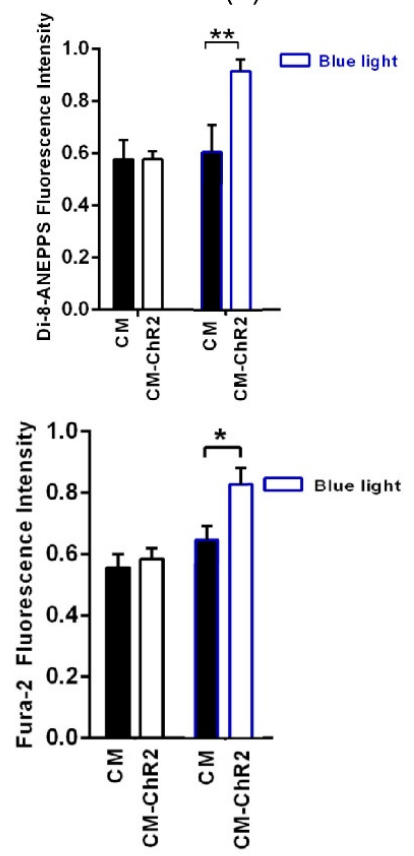

(d)

Figure 3. Electrophysiology Evaluation of CM and CM-ChR2. (a) Fluorescent image of CM-ChR2 labeled by Di-8-ANEPPS in the culture following blue light stimulation. (b) Evaluation of the alteration of transmembrane potential by quantifying Di-8-ANEPPS fluorescence intensity. (c) Fluorescent image of CM-ChR2 loaded by Fura-2 in the culture following blue light stimulation. (d) The fluorescent intensity of Fura-2 was measured, which indicates the intracellular calcium level. 
sensor QuasAr, providing a vigorous tool for evaluating optogenetic pacing and optical electrophysiology simultaneously. ${ }^{10}$ Furthermore, Lapp et al suggest a novel optogenetic recording system analyzing the extracellular field potentials from cardiomyocytes to recognize drug actions at the whole range of physiological cardiac rates. ${ }^{11}$

Optogenetics can be used to induce excitatory membrane currents in specific cell subgroups. Zaglia and colleagues used transgenic mice with specific expression of ChR2 in Purkinje cells and cardiomyocytes to determine the amount of threshold light to stimulate these cells selectively. In fact, the researchers were trying to characterize the level of light energy needed to start an ectopic wave. The results show that the energy required to initiate extraventricular outflow in the left ventricle is 2 to 3.5 times more than the energy needed in the right ventricle or 8 to 20 times in the Purkinje system. ${ }^{12}$

Finally, we provided cardiomyocytes expressing ChR2, which in the future will be helpful not only for the study of electrophysiological properties of the heart but also for the cardiotoxicity prediction of drugs.

\section{Ethical Considerations}

Ethical approval was obtained from the research ethics committee of the Iranian Academic Center for Education, Culture and Research (IR.ACECR.IBCRC.REC.1305.22).

\section{Conflict of Interests}

The authors declare no conflict of interest.

\section{Acknowledgements}

This research was supported by a grant from Medical Laser Research Center, Yara Institute Academic Center for Education, Culture and Research (ACECR) and Research Council of the University of Tehran.

\section{References}

1. Keating MT, Sanguinetti MC. Molecular and cellular mechanisms of cardiac arrhythmias. Cell. 2001;104(4):56980. doi: 10.1016/s0092-8674(01)00243-4.

2. Chen L, Sampson KJ, Kass RS. Cardiac delayed rectifier potassium channels in health and disease. Card Electrophysiol clin. 2016;8(2):307-22. doi: 10.1016/j. ccep.2016.01.004.

3. Agus V, Janovjak H. Optogenetic methods in drug screening: technologies and applications. Curr Opin Biotechnol. 2017;48:8-14. doi: 10.1016/j.copbio.2017.02.006.

4. Pastrana E. Optogenetics: controlling cell function with light. Nat Methods. 2010;8(1):24. doi.org/10.1038/ nmeth.f.323

5. Schneider F, Grimm C, Hegemann P. Biophysics of channelrhodopsin. Annu Rev Biophys. 2015;44:167-86. doi: 10.1146/annurev-biophys-060414-034014.

6. Keshmiri Neghab H, Goliaei B, Saboury AA, Esmaeeli Djavid G, Pornour M, Hong J, et al. Modulation of cardiac optogenetics by vitamin A. Biofactors. 2019;45(6):983-90. doi: 10.1002/biof.1564. Epub 2019 Sep 11.

7. Schimmel KJ, Richel DJ, van den Brink RB, Guchelaar H-J. Cardiotoxicity of cytotoxic drugs. Cancer Treat Rev. 2004;30(2):181-91. doi: 10.1016/j.ctrv.2003.07.003.

8. Menna P, Salvatorelli E, Minotti G. Cardiotoxicity of antitumor drugs. Chem Res Toxicol. 2008;21(5):978-89. doi: 10.1021/tx800002r. Epub 2008 Apr 1.

9. Klimas A, Ambrosi CM, Yu J, Williams JC, Bien H, Entcheva E. OptoDyCE as an automated system for high-throughput all-optical dynamic cardiac electrophysiology. Nat Commun. 2016;7(1):1-12. doi.org/10.1038/ncomms11542

10. Dempsey GT, Chaudhary KW, Atwater N, Nguyen C, Brown BS, McNeish JD, et al. Cardiotoxicity screening with simultaneous optogenetic pacing, voltage imaging and calcium imaging. J Pharmacol Toxicol Methods. 2016;81:240-50. doi: 10.1016/j.vascn.2016.05.003.

11. Lapp H, Bruegmann T, Malan D, Friedrichs S, Kilgus C, Heidsieck A, et al. Frequency-dependent drug screening using optogenetic stimulation of human iPSC-derived cardiomyocytes. Sci Rep. 2017;7(1):1-12. doi: 10.1038/ s41598-020-80763-7.

12. Zaglia T, Pianca N, Borile G, Da Broi F, Richter C, Campione $\mathrm{M}$, et al. Optogenetic determination of the myocardial requirements for extrasystoles by cell typespecific targeting of ChannelRhodopsin-2. Proc Natl Acad Sci U S A. 2015;112(32):E4495-E504. doi: 10.1073/ pnas.1509380112. 